\title{
On the Pedagogical Relevance of Strategic Competence
}

Tahereh Paribakht

This paper is a report on a study concerned with the identification of target language linguistic material essential for the learners' use of communication strategies (CS) in survival situations.

Subjects were 40 adult ESL students and 20 native speakers of English. A conceptidentification task was used to elicit these speakers' CS. Given that the taxonomy of CS developed in the study was based on the type of knowledge utilized by the speakers, it was possible to identify the semantic, as well as the typical syntactic patterns, required for their implementation. These linguistic manifestations of CS can serve as a basis for developing $\mathrm{L} 2$ teaching materials with the aim of preparing L2 leamers to function successfully in problematic communication situations. An appropriate sequence for the presentation of such material is proposed based on the frequency of their application in the negotiation of meaning by the speakers in this study.

While "communicative language teaching" has been an important phrase in language (L2) teaching for some time now, there is little specific research on exactly what is necessary for basic communication. This study seeks to provide data on this question with respect to second language speakers: What kinds of knowledge permit L2 learners to communicate their basic intentions? Are there certain strategies which can help them overcome gaps in their linguistic knowledge?

One of the main communication problems that second-language learners frequently encounter is lexical gaps in their target language (TL) production, particularly in the early stages of L2 learning. Research on learners' L2 production has shown that they use communication strategies (CS) in getting their meaning across when they encounter lexical problems (e.g. Tarone, 1977; Bialystok and Fröhlich, 1980; Paribakht, 1982). CS are, therefore, underlying elements in speakers' attempts to transmit their thoughts to their interlocutors. One question which arises is what the linguistic realizations of these CS are and what learners need to know in order to be able to implement these strategies in the TL. A further consideration is how an inventory of the linguistic material used in CS can be developed, with the aim of preparing learners for success in communication situations.

Providing L2 learners with the core notions and typical syntactic structures derived from the surface realization of CS may help them to over- 
come lexical gaps that call for the use of these strategies in communication situations. This study was an attempt to identify the surface grammar of CS and to consider the implications of this for L2 syllabus design.

\section{SUBJECTS AND PROCEDURES}

There were three groups of 20 adult subjects each: two groups of ESL students in Canada at two distinct levels of proficiency (intermediate and advanced) ${ }^{1}$ and a group of native speakers of English as the comparison group.

To elicit the speakers' CS, an information gap, or more precisely a lexical gap, that would call for the use of CS had to be created between the subjects and their interlocutors. The communicative task designed for the study was a concept-identification task comprising both concrete and abstract nouns. ${ }^{2}$ The task involved oral interaction between the subjects and their native speaker interlocutors.

In the case of concrete nouns, the pictures of the target items and, in the case of abstract nouns, the target words, were put on separate cards and were presented to the subjects in the same order. The subjects were then asked to try to convey each of the concepts to their interlocutors without using the target words. Interaction between the subjects and their interlocutors continued either until the interlocutor identified the target concept, or until one of the parties gave up.

All interactions were tape recorded for later analysis. During all sessions, the experimenter took note of any meaningful gestures the subjects used in communicating the target concepts.

\section{RESULTS}

Subjects' CS were identified, categorized and grouped into four major communicative approaches on the basis of type of knowledge utilized by the speakers for their realizations:

I. Linguistic Approach, which exploited the semantic features of the target concepts.

II. Contextual Approach, which exploited the speakers' contextual knowledge.

III. Conceptual Approach, which exploited the speakers' world knowledge.

IV. Mime, which exploited the speakers' knowledge of meaningful gestures. 
All three subject groups (intermediate ESL, advanced ESL, and native speakers) used all four communicative approaches in communicating both concrete and abstract nouns, and differed only in the use of a few constituent CS of the Contextual Approach, i.e., Idiomatic Transfer, Transliteration of $\mathrm{L} 1$ Idioms and Proverbs, which by definition, could be used only by the ESL groups, and Use of TL Idioms and Proverbs, used by advanced ESL and native speakers (see Appendix for the taxonomy of CS used by the subjects). Thus, the learner groups and native speakers used the same types of CS but differed in the relative frequency of use of the four approaches (see Table 1 and Table 2).

Table 1

Summary for Between Group Differences in the Use of the

Four Major Approaches in the Separate Data

\begin{tabular}{lll}
\hline CS & Concrete Nouns & Abstract Nouns \\
\hline Linguistic Approach & NS, G-A $>$ G-I & G-A, NS $>$ G-I \\
Contextual Approach & n.s. & n.s. \\
Conceptual Approach & G-I $>$ G-A & G-I $>$ G-A, NS \\
Mime & G-I, G-A $>$ NS & n.s. \\
\hline
\end{tabular}

NS = native speakers

$\mathrm{G}-\mathrm{A}=$ advanced group

$\mathrm{G}-\mathrm{I}=$ intermediate group

Newman-Keuls $p<.05$

Table 2

Summary for Between Group Differences in the Use of the Four Major Approaches in the Merged Data

\begin{tabular}{lc}
\hline CS & Concrete Nouns + Abstract Nouns \\
\hline Linguistic Approach & NS, G-A $>$ G-I \\
Contextual Approach & n.s. \\
Conceptual Approach & G-I $>$ G-A, NS \\
Mime & G-I, G-A $>$ NS \\
\hline
\end{tabular}

NS = native speakers

$\mathrm{G}-\mathrm{A}=$ advanced group

$\mathrm{G}-\mathrm{I}=$ intermediate group

Newman-Keuls $\mathrm{p}<.05$ 
On the basis of these results, it appears that adult speakers share a certain ability or "competence" to deal with their communicative problems. This has been referred to as "strategic competence," one of the components of communicative competence in the framework proposed by Canale and Swain (1980). ${ }^{3}$ However, while the speakers in this study had basically the same types of tools, i.e. CS, to cope with lexical gaps in their TL, their ability to access these tools in L2 communication depended on the availability of the types of knowledge outlined above. An increase in the speakers' TL proficiency level simply provided the learners with the type of knowledge, e.g., semantic, they needed to utilize a given strategy; it did not seem to affect their underlying strategic competence. Thus learners' limited (or lacking) TL knowledge may not only preclude (in terms of type), but may also reduce (in terms of frequency) the use of certain CS that require that knowledge. Strategic competence is an ability whose application in the TL becomes possible only if the knowledgelinguistic and otherwise-is available to the learner in the communication situation.

In that the CS taxonomy developed in this study was based on the type of knowledge utilized by the speakers, it provides a basis for identifying the linguistic content of CS, i.e., the semantic components, as well as the typical syntactic structures required for their implementation.

The taxonomy indicates sets of vocabulary items, such as superordinate terms, synonyms and antonyms, that the speakers needed, in the Linguistic Approach, in conveying both concrete and abstract concepts (see Appendix A). In defining concrete nouns (i.e., objects), the speakers referred to their physical properties such as their size, colour and material, and to their locational, spatial, as well as historical and functional properties.

\section{Examples:}

1) "It's small" (pomegranate)

"It would fit into your hand" (pomegranate)

2) "It's usually rounded" (turret)

"It's frilly" (ruff)

3) "It's red" (pomegranate)

4) "It's made of metal" (thimble)

5) "They used to use... wear around their neck" (ruff)

Providing learners with the lexical items needed to express notions such as physical properties should enable them to perform the communicative act of defining a concept. That is, having these basic lexical items at their disposal, learners should be able to define in the TL concrete or abstract nouns in terms of relationships of space, time, etc.

The taxonomy also indicates that subjects gave metalinguistic clues to 
their interlocutors in communicating the target items, for example, "It's actually a noun with a suffix" (martyrdom). Giving learners some metalinguistic knowledge may prove to be valuable to them in their coping with their lexical problems.

The constituent CS of the Contextual Approach indicate that knowledge of TL idioms and proverbs can provide speakers with alternative means to carry through their intended meanings. The instruction of TL idioms and proverbs can be graded, as Yorio (1980) also suggests, through the use of structural, syntactic and semantic criteria. However, the quantitative analysis of the data (See Table 3) showed that the Contextual Approach was least frequently adopted by all subject groups, suggesting that this type of knowledge, although useful, was not as crucial as the speakers' TL linguistic knowledge in conveying their meanings.

Table 3

Frequency Distribution of the Use of Each Major Communicative Approach by Each Group and for Each Item Set

\begin{tabular}{lcccccc}
\hline \multirow{2}{*}{ Approaches } & $\begin{array}{c}\text { Intermediate } \\
(\mathrm{N}=20)\end{array}$ & \multicolumn{2}{c}{$\begin{array}{c}\text { Advanced } \\
(\mathrm{N}=20)\end{array}$} & \multicolumn{2}{c}{$\begin{array}{c}\text { Native } \\
(\mathrm{N}=20)\end{array}$} \\
\cline { 2 - 7 } & $\begin{array}{c}\mathrm{CN} \\
(\mathrm{n}=197)\end{array}$ & $\begin{array}{c}\mathrm{AN} \\
(\mathrm{n}=200)\end{array}$ & $\begin{array}{c}\mathrm{CN} \\
(\mathrm{n}=144)\end{array}$ & $\begin{array}{c}\mathrm{AN} \\
(\mathrm{n}=200)\end{array}$ & $\begin{array}{c}\mathrm{CN} \\
(\mathrm{n}=200)\end{array}$ & $\begin{array}{r}\mathrm{AN} \\
(\mathrm{n}=200)\end{array}$ \\
\hline $\begin{array}{l}\text { Linguistic } \\
\text { Approach }\end{array}$ & 821 & 843 & 627 & 843 & 749 & 671 \\
$\begin{array}{l}\text { Contextual } \\
\text { Approach }\end{array}$ & 6 & 205 & 1 & 153 & 7 & 144 \\
$\begin{array}{l}\text { Conceptual } \\
\text { Approach }\end{array}$ & 47 & 327 & 17 & 192 & 26 & 137 \\
Mime & 74 & 3 & 49 & 1 & 13 & 0 \\
& 948 & 1378 & 694 & 1189 & 795 & 952 \\
\hline
\end{tabular}

$\mathrm{CN}=$ Concrete nouns

AN $=$ Abstract nouns

$\mathrm{n}=$ number of items tried

Both Contextual and Conceptual Approaches give an indication of the importance of the speakers' world knowledge, particularly that which corresponds to TL cultural and social properties. Effective L2 teaching would need to incorporate such information either directly through the 
content of the TL curriculum, or indirectly, through the content of other subject curricula, even if taught in the learner's $\mathrm{L} 1$.

The taxonomy further indicates that paralinguistic knowledge can also be utilized in overcoming lexical gaps in communication. Familiarizing learners with meaningful gestures and facial expressions specific to the target culture may also counteract a possible source of misunderstanding and misinterpretation and may, consequently, contribute to more effective communication.

An appropriate sequence for the presentation of the above elements could be established according to the frequency with which the speakers used them. This study showed that the Linguistic Approach was the approach most frequently adopted by all subject groups, suggesting that the information needed for its adoption was most fundamental for the subjects' communicative survival.

In addition to the components discussed above, the subjects' CS were fairly consistently associated with particular syntactic structures. It is, therefore, possible to compile an inventory of typical sentence patterns which can be converted into $\mathrm{L} 2$ teaching material. The typical sentence patterns embodying the subjects' CS were:

Sentence Pattern

This (it) is...

This (it) has...

This (it) does...

\section{CS}

Superordinate, Synonymy, Antonymy, Size, Material, (active or passive form), Historical Property (active or passive form), Locational Property (active or passive form), Other Features, Metalinguistic Clues (active or passive form)

Features, Elaborated Features

Functional Description (active or passive form).

The comparative form for Analogy and Contrast almost always used the "it is (not) like..." or the "(not) as... as" patterns. Furthermore, the sentence patterns used were expressed in affirmative or negative forms, simple present or past (sometimes simple future) tense and active (sometimes passive) forms. Conditional forms were occasionally used in strategies such as Demonstration for the creation of hypothetical contexts. In general, it appeared that for communicating concrete nouns, sentencelevel grammar and for communicating abstract nouns, discourse-level grammar (i.e., knowledge of cohesion markers and coherence rules) were most relevant. 
Learning these typical syntactic structures and sentence patterns will, therefore, enable learners to express the information utilized in the related strategies. The presentation of these syntactic patterns could be ordered according to the frequency of their applicability and productivity in the learners' negotiation of meaning.

\section{A STRATEGIC FOCUS FOR L2 TEACHING}

All the above components (i.e., lexical, syntactic, idiomatic) constitute what could be referred to (using Allen's (1981) terminology) as the "surface structure grammar" of CS. An L2 curriculum component based on this grammar could be developed, with the aim of preparing L2 learners for the negotiation of meaning in the absence of the target lexical items. This component, which could be referred to as the strategic component, would enable learners to overcome lexical gaps (i.e., concrete nouns and abstract nouns) in communication situations and would respond, therefore, to one of the important and basic communicative needs of L2 learners.

Allen (1983) has proposed a comprehensive three level approach to L2 teaching. His framework includes structural analytic, functional analytic, and experiential components. In the structural analytic component (e.g., Dodson, 1976) goals are defined in terms of grammatical accuracy and the sentence, broken down into its grammatical constituents, is the basic unit of description. In the functional analytical component (e.g., Wilkins, 1976), speech acts constitute the basis for description and learners are taught to produce coherent discourses. The experiential level (Stern, 1979) encourages spontaneous unanalyzed use of language in natural communicative settings. Allen proposes a balance among the three components, and suggests a three-level communicative course in which the relevant knowledge in each component is incorporated. Allen's model could be expanded to include a fourth component, i.e., the strategic component (see Figure 1). The specific features of the strategic component are: first, it takes advantage of a learner's ability (i.e., strategic competence) already available to him/her in the L2 learning situation; second, the learner's needs are not determined or defined by the teacher, but rather are initiated by the learner. There is, therefore, no pre-selection of items. The items are derived from those expressed by the learner in solving communicative problems. Third, the unit of description/instruction is a strategy.

As far as the sequence for presenting the components of the model is concerned, it would seem advantageous to introduce the strategic component early. This would not only establish the fundamental syntactic structures and lexical items needed for the learner's negotiation of meaning, 
but will also start the learner on some communicative skills to be used in extended communicative situations. The more quickly the learners take advantage of what they have learned in this component, the better they will be prepared to cope with some problematic communicative situations.

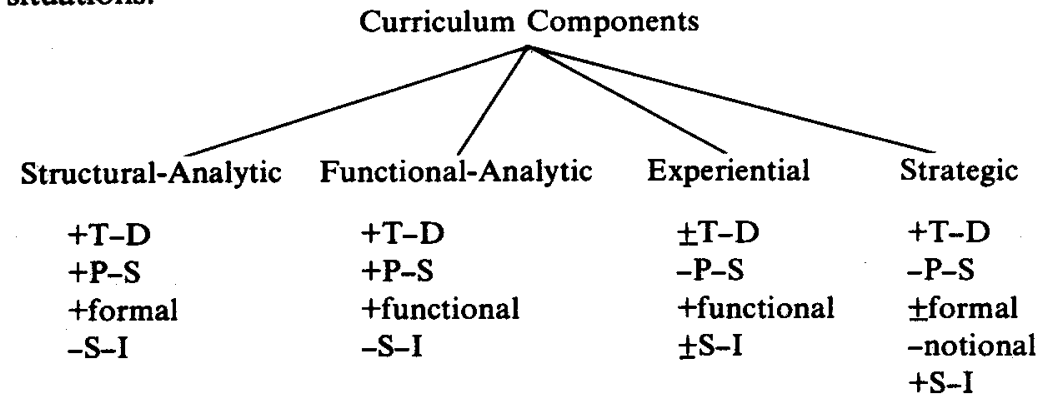

Teacher-directed (T-D)

Pre-selection of items (P-S)

Student-initiated (S-I)

Figure 1. Suggested curriculum components of communicative $\mathbf{L} 2$ teaching

Once learners are provided with such TL materials whose areas of application are clearly definable, they could then be given the opportunities to practice the use of CS in situations where a lexical gap is created between the learner and the interlocutor. Communication tasks such as the one designed for this study (i.e., concept-identification task) or similar ones which involve the creation of information gap between the interlocutors could serve such a purpose. Frequent practice of this kind will make the corresponding linguistic material more readily accessible to the learners, and they can benefit from this in real communication situations inside and outside the classroom. Such practice may also promote learners' flexibility and enable them to integrate all their knowledge sources and use alternative means in transmitting their intended meanings.

The proposed strategic component certainly requires further work and testing. At this stage of our knowledge, this component cannot be an end in itself but rather is complementary to the instructional goals of a multi-component communicative approach to L2 teaching. Further research on other aspects of the speaker's strategic competence (e.g., written production strategies, reception strategies-aural and written) and on strategies associated with production problems other than lexical (e.g., syntactic or sociolinguistic), could provide an extensive inventory of strategies as a basis for the development of a comprehensive L2 curriculum. 
In summary, while in this paper the speakers' CS were analyzed for basic lexicon and surface grammar and some suggestions were made for the incorporation of the identified items into an $L 2$ teaching curriculum, the main purpose of the paper was not to provide an exhaustive inventory of such items, but was rather to propose a framework for the identification of some basic needs of $\mathrm{L} 2$ learners in overcoming lexical gaps in communication situations.

\section{NOTES}

1. The grammatical proficiency level of the subjects was measured by the Michigan Test of English Language Proficiency. The oral proficiency levels of the groups were then determined by the IEA (International Educational Achievement) Test of Proficiency in English as a Foreign Language.

2. Examples of the items used in the study are as follows:

\begin{tabular}{lll}
\multicolumn{2}{c}{ Concrete Nouns } & Abstract Nouns \\
turret & abacus & fate \\
aqueduct & hammock & martyrdom \\
ruff & lantern & flattery \\
pillory & scarecrow & success \\
yoke & seesaw & honesty \\
decanter & funnel & pride \\
trolley & thimble & courage \\
cherub & pomegranate & faithfulness \\
cruet-stand & dust pan & justice \\
& palanquin & patience
\end{tabular}

3. Canale and Swain's (1980) model of Communicative Competence includes three main components: grammatical competence (the knowledge of the grammatical rules of the target language), sociolinguistic competence (the knowledge of the social rules of language use) and strategic competence (the knowledge of verbal and non-verbal communication strategies).

\section{REFERENCES}

Allen, J.P.B. (1983). Three level curriculum model for L2 education. Canadian Modern Language Review, 40(1), Oct.1983, 23-43.

Allen, J.P.B. (1981). The audiolingual method: where did it come from and where is it going? Manuscript. Toronto: OISE.

Bialystok, E. and Frölich, M. (1980). Oral communication strategies for lexical difficulties. Interlanguage Studies Bulletin. 5(1), 3-30.

Canale, M. and Swain, M. (1980) Theoretical bases of communicative approaches to second language teaching and testing. Applied Linguistics, $1(1), 1-4$.

Dodson, C.J. (1976). Foreign language teaching and bilingualism. Bilingualism and British education: The Dimensions of diversity. CILT Reports and Papers 14. London: Centre for Information and Language Teaching and Research. Paribakht, T. (1982). The relationship between the use of communication strategies and aspects of target language proficiency: A study of Persian ESL students. Ph.D. thesis, University of Toronto. 
Paribakht, T. (1985). Strategic competence and language proficiency. Applied Linguistics. 6(2), 132-146.

Stern, H.H. (1979) Some approaches to communicative Language Teaching in Canada. Paper presented at the ACTFL European American Seminar, New York, 9-12 October, 1979.

Tarone, E. (1977) Conscious communication strategies in interlanguage: a progress report. In D. Brown, C. Yorio and R.M. Crymes (Eds.), On TESOL '77. Washington, D.C.: TESOL.

Wilkins, D.A. (1976). Notional syllabuses. London: Oxford University Press. Yorio, C. (1980). Conventionalized language forms and the development of communicative competence. TESOL Quarterly, 14(4), 433-442.

\section{THE AUTHOR}

Tahereh Paribakht teaches ESL and Applied Linguistics at the University of Ottawa, Centre for Second Language Learning and the Faculty of Education. She has a Ph.D. in Applied Linguistics from the University of Toronto, the Ontario Institute for Studies in Education (OISE). For the past several years she has been investigating the notion of strategic competence in second language communication. 


\section{APPENDIX \\ TAXONOMY OF COMMUNICATION STRATEGIES*}

\section{Linguistic approach}

This approach exploits the semantic features of the target item and reflects the speaker's formal analysis of meaning.

A. Semantic contiguity. All CS in this category exploit items semantically related to the target item.

1. Superordinate. For example:

'This is a fruit' (pomegranate)

'This is a quality' (honesty)

2. Comparison. This is the strategy of exploiting similarities between the two items.

a. Positive comparison

i. Analogy. For example:

'Is the same like lamp' (lantern)

'It is like the victory' (success)

ii. Synonymy. For example:

'Caravan' (palanquin)

'Synonym for wait' (patience)

b. Negative comparison

i. Contrast and opposition. For example:

'It's not a same as computer' (abacus)

'When you don't have it, you're scared' (courage)

ii. Antonymy. For example:

'This is the opposite of failure' (success)

'Opposite it's is exactly hurry' (patience)

B. Circumlocution. This strategy, which is an attempt to describe the characteristics of the concept, includes:

1. Physical description.

a. Size. For example:

'It would fit into your hand' (pomegranate)

b. Shape. For example:

'This fruit have a shape like earth' (pomegranate)

c. Colour. For example:

'Its colour is red' (pomegranate)

d. Material. For example:

'It's made of metal' (thimble)

* This taxonomy appears in Paribakht (1985). 
2. Constituent features. In concrete nouns, constituent features refer to different parts of the object; and in abstract nouns they are the underlying semantic elements of the concept.

a. Features. For example:

'There is a handle on it' (lantern)

'Someone who dies for a cause' (martyrdom)

a. Elaborated features. The details of a single feature of the item are given. For example:

'has always little juicy seeds inside and they are red, and they're really tart' (pomegranate)

'being killed in, usually in-for a good cause' (martyrdom)

3. Locational property. For example:

'It was used maybe in Arab countries' (palanquin)

'Tie with two, two trees, we tie to two trees' (hammock)

4. Historical property. For example:

'It belong to many many years ago' (abacus)

'Ancient people used this' (palanquin)

5. Other features. Other features refer to those features which are not necessarily factual, but rather are indirectly associated with the target items. While some of these associations may be shared by speakers of different linguistic backgrounds (see the first example below), many of these specific associations appear to be contextand/or culture-bound (see the second example below).

'It's workmate to a broom' (dust-pan)

'It's the passion fruit' (pomegranate)

'It's honourable' (martyrdom)

6. Functional description. For example:

'When you finish sweep-ah-you use-you used for collect garbage' (dust-pan)

C. Metalinguistic clues. The speaker gives metalinguistic information on the target item. For example:

'It's actually a noun with a suffix' (martyrdom)

\section{Contextual approach}

This approach exploits the contextual knowledge of the speaker. That is, it provides contextual information about the target item rather than its semantic features.

A. Linguistic context. This is the strategy of providing a linguistic context for the target item, leaving the target item blank. Examples:

'When you sweep the floor, you gather up the dust with (dust-pan)

'If the wife fools around with somebody else, she is not this to the husband' (faithfulness) 
B. Use of target language idoms and proverbs. This strategy exploits one's knowledge of target idioms or proverbs to refer the interlocutor to a specific and popular context where the target item is used. Examples: 'It comes before a fall' (pride)

'It gets you nowhere' (flattery)

C. Transliteration of $\mathrm{Ll}$ idioms and proverbs. The speaker attempts to translate an $\mathrm{L} 1$ idiom or proverb into the target language. Examples: 'Some say, it's written on your forehead' (fate)

'When somebody is so good-the heart is so clean' (honesty). (In Farsi, a 'clean-hearted person' refers to an honest person.)

D. Idiomatic transfer. This strategy involves reference to some semantic or syntactic feature of an Ll idiom, as opposed to its actual translation, assuming that it will work the same way in the target language. Examples:

'I take an examination and I fail, O.K.? and one of my adjectives has been broken' ('to break one's pride')

'You say, O.K. "good luck”. What's another word for "good luck”?' (success). (The subject has considered Persian 'be successful' as a synonym for its corresponding expression in English, 'good luck'.)

III Conceptual approach

The conceptual approach exploits the speaker's knowledge of the world and of particular situations. This knowledge may be biased or influenced by the speaker's social and/or cultural background.

A. Demonstration. This is the strategy of creating a concrete context that reflects the target concept. Examples:

'Suggest that you are a teacher and I am a student; and I didn't take the-for-pass and I fail; and I come and say something, for example, you teach very well, you are a good man and-what's the name of my action?" (flattery)

B. Exemplification. This is the strategy of reference to examples, such as certain people, occasions, or real events, that correspond to the target concept. Examples:

'You may use it in camping' (lantern)

'A soldier in a war definitely needs it' (courage)

'The servants especially do, for example, to their masters' (flattery)

C. Metonymy. The concept is represented through a prototype member of that concept which may or may not be shared by different cultures and speech communities. Examples:

'It's symbolized by a dog' (faithfulness)

'peacock' (pride)

IV Mime

This non-verbal strategy refers to the use of meaningful gestures in com- 
municating the target item. Mime has the following sub-categories:

A. Replacing verbal output. This non-linguistic strategy is used by the speaker to substitute for a linguistic output. Examples:

'It's this size' (pomegranate)

'You always think are higher than me and you look me like this' (mime for a snobbish look) (pride)

B. Accompanying verbal output. In adopting this paralinguistic strategy, the speaker uses a meaningful gesture to accompany his or her verbal output. Examples:

'It goes up and down' (mime for the movement) (seesaw)

'This fruit have a shape like earth' (mime for a round shape) (pomegranate) 